\title{
UM MODELO DE PROGRAMAÇÃO LINEAR INTEIRA PARA OTIMIZAÇÃO DE RECURSOS NA DESIGNAÇÃO DE ÁRBITROS PARA O CAMPEONATO BRASILEIRO DE FUTEBOL ${ }^{1}$
}

\author{
Iago Cambre Añon ${ }^{\text {a*}, ~ A l c i d e s ~ J o s e ́ ~ S c a g l i a a ~}{ }^{\text {a }}$ Cristiano Torezzan ${ }^{a}$

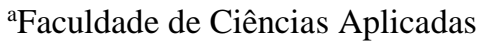 \\ Universidade Estadual de Campinas - UNICAMP, Limeira-SP, Brasil
}

Recebido 06/03/2016, aceito 16/11/2016

\begin{abstract}
RESUMO
Este trabalho apresenta um modelo de Programação Linear Inteira para a designação de árbitros para partidas em campeonatos de futebol. A função objetivo minimiza a distância total percorrida pelos árbitros e as restrições modelam as preferencias e normas definidas pelos responsáveis pela escala. O modelo foi testado com dados reais do Campeonato Brasileiro de Futebol da Série A de 2014 e os resultados mostram que, utilizando a proposta deste artigo, seria possível economizar até 38,25\% na distância total percorrida pelos árbitros e, ainda, respeitar um limite máximo de duas partidas do mesmo árbitro para cada time e um total entre dezoito e vinte partidas por árbitro no campeonato. O modelo proposto pode ser adaptado para incorporar diferentes regras ou preferências ou, ainda, ser utilizado para escalas de árbitros em outros esportes.
\end{abstract}

Palavras-chave: Designação de árbitros, Futebol, Programação linear inteira, Esporte.

\begin{abstract}
We consider an Integer Linear Model to address the referee assignment problem. The objective function is to minimize the total distance traveled by the referees while dealing with rules and policies requested by the decision maker. The model was tested with real data from the first league Brazilian championship of 2014 and the results show that using our approach it is possible to reduce up to $38.25 \%$ in the total distance traveled by the referees and also limiting in two matches by referee for each team as well as balancing the number of assigned matches for each individual referee during the tournament. The proposed model may be adapted to incorporate different preferences or even be used for referee assignment in other sports.
\end{abstract}

Keywords: Referee assignment, Football, Integer linear programming, Sports.

*Autor para correspondência. E-mail: iago.anon@gmail.com 


\section{Introdução}

Ligas profissionais de futebol são grandes negócios no mundo e movimentam um volume significativo de recursos financeiros (Recalde et al., 2013). Diariamente, milhares de torcedores acompanham jornais, rádio, televisão e internet em busca de informações sobre times locais, nacionais e internacionais, além de resultados e estatísticas sobre jogos, campeonatos e jogadores (Ribeiro e Urrutia, 2012).

Neste esporte, a função dos árbitros envolve garantir que todas as regras sejam respeitadas e que a partida seja conduzida de forma ética, privilegiando o desenvolvimento do jogo como um espetáculo esportivo (Duarte et al., 2007). Neste cenário, a atuação dos árbitros é alvo frequente de grande pressão por parte dos diversos atores envolvidos (clubes, atletas, torcedores, mídia). Diversos fatores podem influenciar na atuação de um árbitro em uma partida, mas os aspectos técnicos e logísticos da profissão têm recebido maior atenção das confederações e entidades de classe. Há uma demanda crescente pela profissionalização dos árbitros, assim como já ocorreu em outros setores do futebol.

Neste contexto, a designação de árbitros para as partidas é uma decisão complexa e de grande responsabilidade, em especial em campeonatos longos e em territórios extensos, como é o caso do Campeonato Brasileiro de Futebol da Série A. Além disso, em função de sua popularidade e do volume de negócios envolvidos, o futebol é um esporte bastante visado quando se trata de corrupção de resultados. Embora exista bastante preocupação com a lisura esportiva, escândalos como o "Calciopoli" no futebol italiano (Distaso et al., 2012) é um exemplo onde árbitros foram envolvidos em casos controversos de resultados (Alarcón et al., 2014).

Assim, o desenvolvimento de políticas mais apropriadas e eficientes para a escolha de árbitros é fundamental para o desenvolvimento do esporte, tanto do ponto de vista técnico quanto para um gerenciamento mais transparente das escalas de arbitragem.

No entanto, na prática, a designação de árbitros na maioria dos campeonatos profissionais não evoluiu na mesma proporção que outras áreas, como, por exemplo medicina esportiva, treinamento e marketing, que incorporaram uma cultura científica para abordar seus principais problemas. Ao contrário, não é raro de se encontrar campeonatos onde a escala de árbitros é feita sem critérios analíticos claros, dando margem a interferências subjetivas indesejáveis ao esporte.

O problema de escala ou designação dos árbitros tem recebido atenção da comunidade científica, em especial da área de pesquisa operacional, pois sua solução manual além de ineficaz é vulnerável a especulações (Kendall et al., 2010 e Ribeiro, 2012). O estudo de Yavuz et al. (2008) propôs um modelo que foi utilizado para identificar uma atribuição justa dos árbitros de futebol para a temporada do campeonato turco da primeira divisão 2005-2006. Ainda neste contexto, Trick e Yildiz (2007) apresentam um modelo com intuito de minimizar a distância total percorrida pelos árbitros.

Neste trabalho, utilizamos um modelo similar ao proposto em Alarcón et al. (2014) para a designação de árbitros para jogos de futebol que utiliza programação linear inteira. O objetivo específico deste artigo é propor e aplicar um modelo para a designação de árbitros utilizando dados reais do Campeonato Brasileiro de 2014, com o intuito de minimizar a distância total percorrida pelos árbitros. A formulação proposta evita que o mesmo árbitro apite várias partidas de um time e ainda propõe uma distribuição uniforme no número total de jogos atribuídos a cada árbitro na competição. Além destes aspectos, o modelo permite ainda a inclusão de outras restrições que podem surgir na prática, como por exemplo, um determinado árbitro não ser elegível para apitar um determinado jogo.

O restante do trabalho está organizado da seguinte forma: na Seção 2, apresentamos a definição do problema e alguns dados do campeonato de 2014. Na Seção 3, apresentamos e discutimos o modelo que será utilizado. A Seção 4 apresenta os resultados computacionais e, por fim, na Seção 5, trazemos as considerações finais. 


\section{Definição do Problema}

O Campeonato Brasileiro de Futebol da Série A, também conhecido como Brasileirão, possui 20 equipes que se enfrentam na modalidade todos contra todos, em partidas no formato turno e returno. O Campeonato é dividido em 38 rodadas de 10 partidas, totalizando 380 jogos. $\mathrm{Na}$ edição de 2014 da competição foram utilizados um total de 59 árbitros, sendo que 39 árbitros apitaram menos de 8 jogos no ano. Como apresentaremos na Seção 5, apenas 20 árbitros seriam suficientes para uma escala racional de recursos.

Sendo o Brasil um país de grandes dimensões geográficas, uma das preocupações que devem ser consideradas na designação dos árbitros é justamente a distância percorrida nas viagens para apitar as partidas. O gerenciamento não adequado desta característica pode aumentar os gastos financeiros com deslocamento e levar a um desgaste físico dos árbitros, afetando seu desempenho técnico no jogo.

O modelo proposto neste trabalho pretende minimizar a soma de todas as distâncias percorridas pelos árbitros para apitar as partidas. Para viabilizar a coleta de dados, consideramos que a distância percorrida por um árbitro para apitar uma partida é igual a distância, em linha reta, entre a capital do estado cujo árbitro reside e a capital do estado onde o jogo foi realizado. Esta distância é multiplicada por 2 para computar a viagem de ida e volta. No exemplo apresentado neste artigo, os dados foram coletados pelos autores utilizando a ferramenta computacional disponível no website http://distanciacidades.com/.

Com base nestas distâncias, foi possível concluir que, para o Campeonato Brasileiro de Futebol da Série A de 2014, os árbitros percorreram um total de 833.516 quilômetros em viagens para apitar todas as partidas.

Outro fator que foi considerado no modelo de designação de árbitros é o número de partidas que um mesmo árbitro apita de uma dada equipe. A repetição de um mesmo árbitro para diversos jogos de uma equipe pode prejudicar a imparcialidade na condução das partidas, além de levantar dúvidas sobre a lisura de resultados. Desta forma, quanto menor for o número de partidas de uma mesma equipe comandadas por um dado árbitro, melhor será o processo de designação.

Na Figura 1 apresentamos o número máximo de partidas designadas à um mesmo árbitro no Campeonato Brasileiro de 2014 - Série A. Pode-se notar que apenas Chapecoense e Criciúma tiveram no máximo 2 partidas atribuídas ao mesmo árbitro, enquanto 4 equipes tiveram cinco partidas apitadas pelo mesmo árbitro.

Figura 1. Número máximo de partidas designadas à um mesmo árbitro no Campeonato Brasileiro de 2014 - Série A.

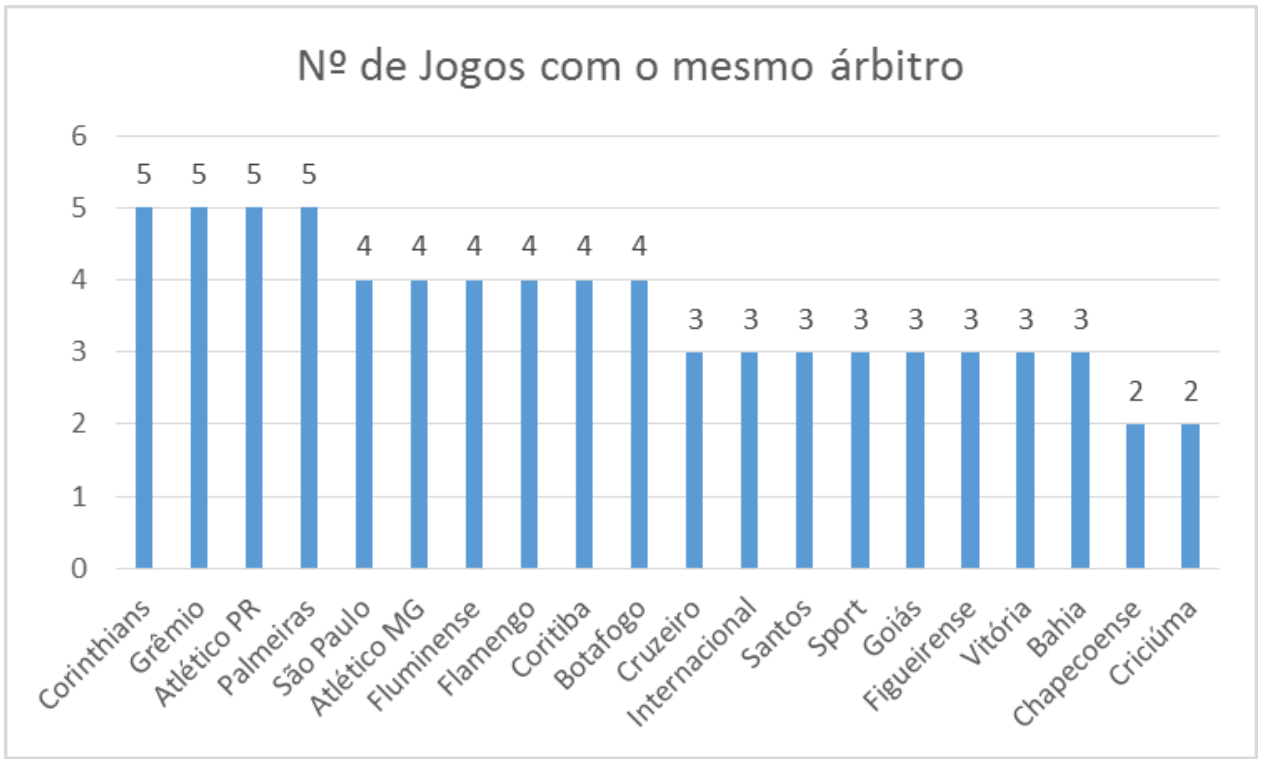

Fonte: Dados coletados das súmulas oficiais disponíveis no site da CBF. 
Visando obter uma maior homogeneidade na designação vamos considerar também o número total de partidas atribuídas para cada árbitro no campeonato.

Na Figura 2 apresentamos o número de partidas apitadas pelos 20 (dos 59) árbitros que mais atuaram no Campeonato Brasileiro de 2014. Pode-se notar que a diferença entre o árbitro que mais apitou (24) e o que menos apitou (8) é de 16 partidas. Se incluirmos os 59 árbitros a diferença passa a ser de 23 partidas.

Figura 2. Número de partidas para cada um dos 20 árbitros que mais atuaram no Campeonato Brasileiro de 2014.

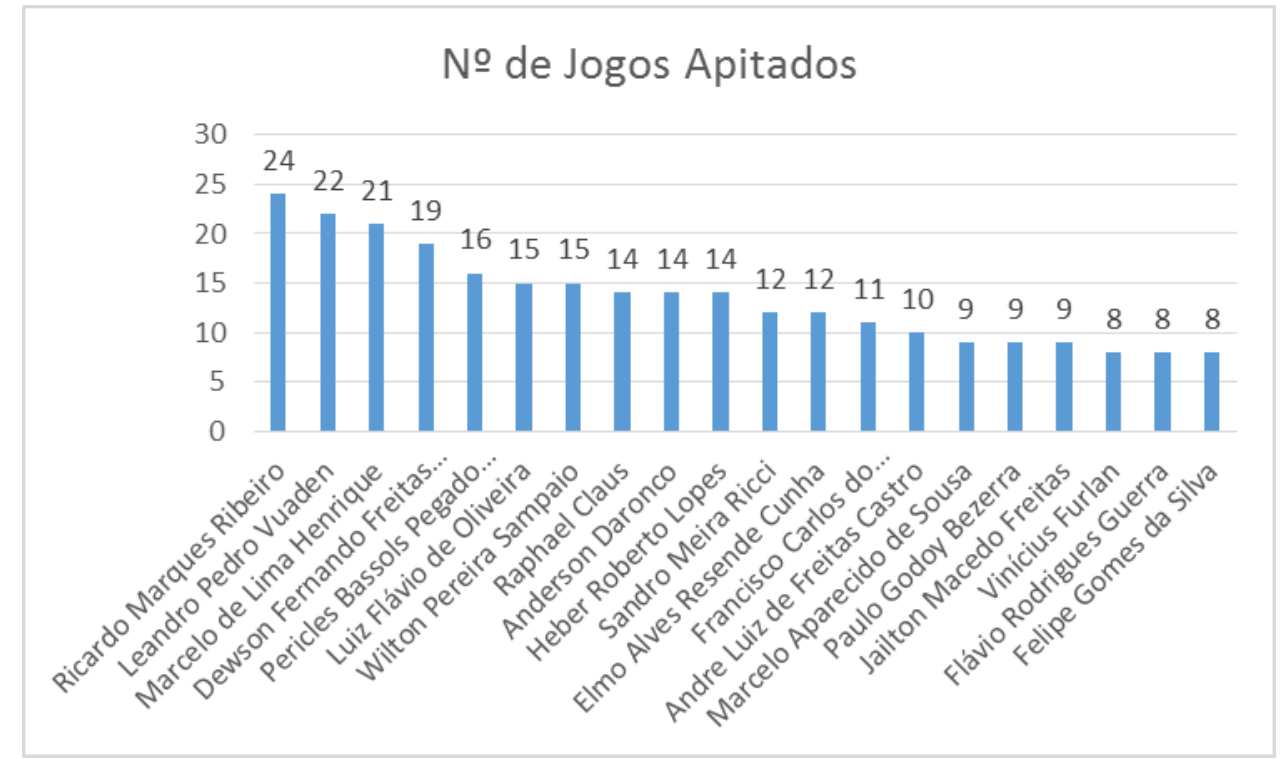

Fonte: Dados coletados das súmulas oficiais disponíveis no site da CBF.

O problema de otimização considerado neste trabalho consiste em minimizar a distância total percorrida pelos árbitros para apitar todas as partidas do Brasileirão, não permitindo que o mesmo árbitro apite mais do que 2 partidas de uma mesma equipe e que a diferença entre o número total de partidas apitadas por cada árbitro no campeonato seja no máximo 2.

Uma questão que surge naturalmente da análise do problema acima é qual é o número mínimo de árbitros necessários para obter-se uma escala factível que atenda os critérios estabelecido? Como são 38 jogos de cada equipe e cada árbitro pode apitar no máximo 2 desses jogos, são necessários no mínimo 19 árbitros para atender esta restrição. Obviamente, quanto mais árbitros estiverem disponíveis, menor será a distância total a ser percorrida.

Neste trabalho, fixamos o número de 20 árbitros para efeito de comparação com os dados reais. Sendo assim, consideramos os 20 árbitros que mais atuaram no Campeonato Brasileiro de 2014 e definimos a capital de seus estados de origem como ponto inicial da viagem para apitar cada partida. $\mathrm{O}$ nome dos árbitros considerados, bem como o número de partidas que cada um apitou no Brasileirão de 2014 estão apresentados na Figura 2.

\section{Modelo Proposto}

O modelo que utilizaremos neste trabalho é baseado em Alarcón et al. (2014) e propõe uma adequação do problema clássico de designação para atender as características peculiares deste caso.

De forma sucinta, o objetivo definir uma escala de árbitros para todo o Campeonato de forma a minimizar a soma das distâncias percorridas pelos árbitros em viagens para apitar os jogos, sujeito à um conjunto de restrições que garantam um número máximo de partidas de um árbitro com a mesma equipe bem como limites mínimos e máximos para o total de jogos de um mesmo árbitro no campeonato.

Para especificação do modelo vamos definir a seguir os seguintes conjuntos: 
- $\mathrm{A}=\{1,2, \ldots, 20\}$ : Árbitros;

- $\mathrm{E}=\{1,2, \ldots, 20\}:$ Equipes;

- $\mathrm{P}=\{1,2, \ldots, 380\}$ : Partidas;

- $\mathrm{R}=\{1,2, \ldots, 38\}$ : Rodadas.

O modelo terá como input os seguintes parâmetros:

- $d_{p, a}$ : distância entre o local da Partida p para o local de origem do Árbitro a

- $\quad \alpha_{p, r}=\left\{\begin{array}{c}1 \text { se a Partida p pertence a Rodadar } \\ 0 \text { caso contrário }\end{array}\right.$

- $\quad \beta_{p, e}=\left\{\begin{array}{c}1 \text { se a Partida } p \text { contém a Equipe e } \\ 0\end{array}\right.$

- $\quad \operatorname{Min}_{a}$ : Número mínimo de partidas a serem apitadas por um árbitro = 18;

- $\operatorname{Max}_{a}$ : Número máximo de partidas a serem apitadas por um árbitro = 20;

- $\gamma_{a, e}$ : Número mínimo de partidas de um árbitro com o mesmo clube $=1$;

- $\bar{\gamma}_{a, e}:$ Número máximo de partidas de um árbitro com o mesmo clube $=2$;

As variáveis de decisão do modelo são as seguintes:

- $\quad x_{p, a}=\left\{\begin{array}{c}1 \text { se a Partida } p \text { é apitada pelo Árbitro } a \\ 0, \quad \text { caso contrário }\end{array}, \forall p \in P e \forall a \in A\right.$

Sendo assim, podemos escrever a função objetivo que deve ser minimizada como (1):

$$
\sum_{\substack{p \in P \\ a \in A}} x_{p, a} * d_{p, a}
$$

Para garantir que exatamente 1 árbitro seja designado para cada partida devemos incluir a seguinte restrição (2):

$$
\sum_{a \in A} x_{p, a}=1, \forall p \in P
$$

Como cada árbitro pode ser escolhido para apitar no máximo 1 jogo por rodada, devemos ter (3):

$$
\sum_{p \in P} \alpha_{p, r} * x_{p, a} \leq 1, \forall r \in R, \forall a \in A
$$

A restrição a seguir garante que o número total de partidas apitadas pelo mesmo árbitro esteja no intervalo pretendido (4).

$$
18 \leq \sum_{p \in P} x_{p, a} \leq 20, \forall a \in A
$$

Por fim, as duas últimas restrições restringem o número de jogos atribuídos à um mesmo árbitro para uma dada equipe (5).

$$
1 \leq \sum_{p \in P} \beta_{p, e} * x_{p, a} \leq 2
$$


Com o objetivo de validar o modelo apresentado nesta seção e comparar a solução obtida com um cenário real, implementamos o problema de programação proposto em linguagem MPL e utilizamos o solver GLPK para resolve-lo. Os testes foram executados em um computador Sony Vaio, com processador Intel Core i5-3210M, CPU @2.50GHz, com 6 GB de memória RAM, com sistema operacional Windows 10. Os resultados obtidos estão apresentados na Seção 4.

\section{Resultados}

Utilizando os dados reais do Campeonato Brasileiro de Futebol de 2014 - Série A e considerando apenas os árbitros listados na Figura 2, o modelo de programação linear inteira proposto na Seção 3 foi resolvido de forma exata, com tempo médio de 45 minutos. Este tempo de solução é perfeitamente aceitável, visto que a programação da escala de árbitros pode ser feita (e inclusive refeita) com vários dias de antecedência.

O modelo implementado encontrou o ótimo global da função objetivo, atendendo todas as restrições, com um total de distância percorrida de $514.692 \mathrm{Km}$ para cobrir os 380 jogos. Este número representa uma redução de $38 \%$ em comparação com a distância total percorrida pelos árbitros na competição de 2014. A título de comparação, na competição de 2014 os 20 árbitros considerados neste trabalho apitaram 261 jogos (Figura 2) e, apenas para apitar esses jogos, percorreram $605.420 \mathrm{Km}$, enquanto que o resultado da nossa otimização prevê um total de $514.692 \mathrm{Km}$ para cobrir os 380 jogos do campeonato.

Vale ressaltar que, se considerássemos os 59 árbitros no modelo, a distância total seria, potencialmente, reduzida pela relaxação do espaço de busca.

Figura 3. Distância total percorrida (em Km) pelos árbitros no Campeonato Brasileiro de 2014 Série A em comparação com o resultado da otimização.



Fonte: Dados coletados das súmulas oficiais disponíveis no site da CBF.

Em relação ao número total de partidas designadas para cada árbitro na competição, a Figura 4 mostra que todos os valores ficaram dentro do limite estabelecido de 18 a 20 partidas. Este resultado é muito mais homogêneo do que o ocorrido no campeonato de 2014, cujo intervalo variou de 8 a 24 partidas (Figura 2). 
Figura 4. Número total de partidas atribuídas a cada árbitro pelo modelo.

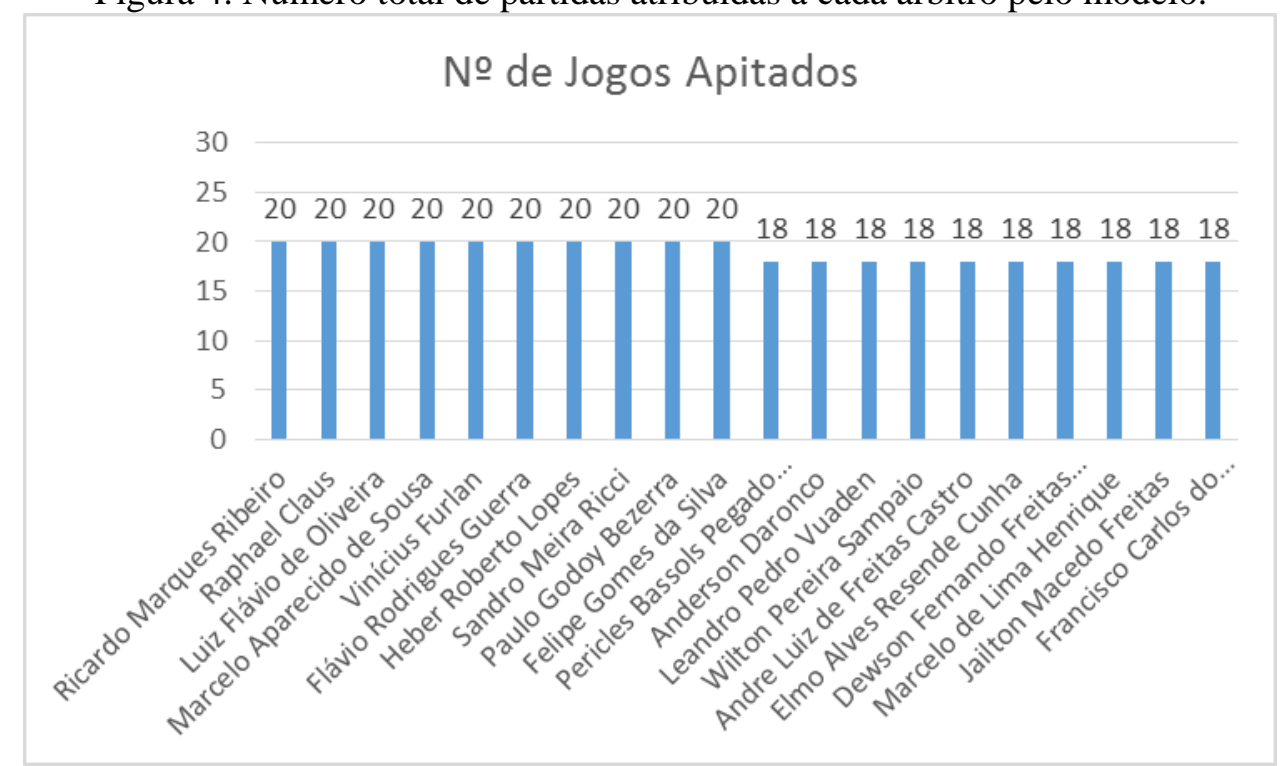

Fonte: Própria - gráfico gerado a partir de resultados do modelo.

Os resultados apresentados nesta seção consideram apenas as restrições que foram inicialmente assumidas. No entanto, o modelo proposto neste trabalho permite que outras restrições sejam incorporadas. Por exemplo, se um determinado árbitro (ou conjunto de árbitros) não puderem apitar uma determinada partida (ou conjunto de partidas) basta zerar as variáveis correspondentes, adicionando restrições do tipo $x_{p, a}=0$. De forma análoga, se for desejado impor um determinado árbitro para uma partida, basta atribuir $x_{p, a}=1$, para o caso correspondente. Outras considerações que podem ser incorporadas, caso seja de interesse da organização, consiste em incluir um número mínimo, $\mathrm{k}$, de jogos apitados por árbitros de um dado nível (ex: árbitros FIFA) para cada equipe. Para tanto pode se incluir uma restrição do tipo (6):

$$
k \leq \sum_{p \in F} \beta_{p, e} * x_{p, a}
$$

Onde $F C P$, representa o subconjunto de árbitro pertencente a categoria que se deseja destacar. Além disso, é possível também flexibilizar o número de jogos por árbitros, ou ainda definir uma função objetivo que seja diferente de minimizar a distância, de forma a adequar-se aos interesses da organização.

\section{Conclusões}

Este trabalho apresenta um modelo de programação linear inteira para designação de árbitros em Campeonatos de Futebol. A função objetivo consiste na minimização da distância total percorrida, atendendo critérios pré-definidos de homogeneidade na escala.

O modelo foi testado com dados reais do Campeonato Brasileiro de Futebol - Série A de 2014 e mostrou que, utilizando apenas 20 árbitros é possível obter uma redução de $38 \%$ na distância total percorrida. Simultaneamente, homogeneizamos o número de partidas atribuídas a cada árbitro.

O modelo proposto permite, ainda, a flexibilização de parâmetros como o número mínimo e máximo de jogos por árbitro e número de partidas no campeonato, bem como a inclusão de novas restrições a critério da organização. Por exemplo, se a comissão de arbitragem 
decidir fixar um determinado árbitro $\varphi$ para uma partida $\rho$, basta apenas incluir a restrição $x_{\rho, \varphi}=1$ e rodar o modelo novamente. Além disso, a função objetivo pode ser adaptada para atender outros interesses, e não apenas a distância percorrida pelos árbitros em viagens, por exemplo, aspectos técnicos e níveis de dificuldades das partidas.

Conclui-se com isso que o modelo proposto pode ser utilizado como apoio a decisão para o atual cenário de designação dos árbitros e é escalável a novas competições e também diferentes modalidades que não foram consideradas neste estudo.

Agradecimentos. Os autores agradecem aos revisores pelas valiosas sugestões e observações que ajudaram a melhorar substancialmente a versão final desse artigo.

\section{Referências}

Alarcón, F., Durán, G. e Guajardo, M. Referee assignment in the Chilean football league using integer programming and patterns. International Transactions in Operational Research, v. 21, n. 3, p. 415-438, 2014.

Distaso, W., Leonida, L., Patti, D. M. A. e Navarra, P. Corruption and referee bias in football: the case of Calciopoli. 2012. Disponível em: SSRN https://ssrn.com/abstract=2004385. Acesso em: 24/08/2016.

Duarte, A. R., Ribeiro, C. C., Urrutia, S. e Haeusler, E. H. Referee assignment in sports leagues. In: Burke, E. K. e Rudová, H. (Eds.) Practice and Theory of Automated Timetabling VI. Springer, 2007. p.158-173.

Kendall, G., Knust, S., Ribeiro, C. C. e Urrutia, S. Scheduling in sports: An annotated bibliography. Computers \& Operations Research, v. 37, n. 1, p. 1-19, 2010.

Recalde, D., Torres, R. e Vaca, P. Scheduling the professional Ecuadorian football league by integer programming. Computers \& Operations Research, v. 40, n. 10, p. 2478-2484, 2013.

Ribeiro, C. C. Sports scheduling: Problems and applications. International Transactions in Operational Research, v. 19, n. 1-2, p. 201-226, 2012.

Ribeiro, C. C. e Urrutia, S. Scheduling the Brazilian soccer tournament: Solution approach and practice. Interfaces, v. 42, n. 3, p. 260-272, 2012.

Trick, M. A. e Yildiz, H. Bender's cuts guided large neighborhood search for the traveling umpire problem. In: Van Hetenryck, P. e Wolsey, L. (Eds.) Integration of AI and OR techniques in constraint programming for combinatorial optimization problems. Springer, 2007. p.332345 .

Yavuz, M., İnan, U. H. e Fiğlalı, A. Fair referee assignments for professional football leagues. Computers \& Operations Research, v. 35, n. 9, p. 2937-2951, 2008. . 